\title{
RNF216 contributes to proliferation and migration of colorectal cancer via suppressing BECN1-dependent autophagy
}

\author{
Hui Wang ${ }^{1, *}$, Yanan Wang ${ }^{1, *}$, Liu Qian ${ }^{1, *}$, Xue Wang ${ }^{1}$, Hailiang Gu ${ }^{2}$, Xiaoqiang Dong ${ }^{2}$, \\ Shiqian Huang ${ }^{1}$, Min Jin ${ }^{1}$, Hailiang $\mathbf{G e}^{1}$, Congfeng $\mathbf{X u}^{1}$, Yanyun Zhang ${ }^{1}$ \\ ${ }^{1}$ Shanghai Institute of Immunology, Institutes of Medical Sciences, Shanghai Jiao Tong University School of Medicine \\ (SJTUSM) and Key Laboratory of Stem Cell Biology, Institute of Health Sciences, Shanghai Institutes for Biological Sciences, \\ Chinese Academy of Sciences \& SJTUSM, Shanghai, China \\ ${ }^{2}$ Department of General Surgery, The First Affiliated Hospital of Soochow University, Suzhou, China \\ *These authors have contributed equally to this work \\ Correspondence to: Yanyun Zhang, email: yyzhang@sibs.ac.cn \\ Congfeng Xu, email: cxu@shsmu.edu.cn \\ Hailiang Ge, email: ghl@shsmu.edu.cn
}

Keywords: RNF2 16, BECN1, autophagy, colorectal cancer

Received: January 29, $2016 \quad$ Accepted: April 28, 2016

Published: May 18, 2016

\section{ABSTRACT}

Originally identified as an E3 ligase regulating toll-like receptor (TLR) signaling, ring finger protein 216 (RNF216) also plays an essential role in autophagy, which is fundamental to cellular homeostasis. Autophagy dysfunction leads to an array of pathological events, including tumor formation. In this study, we found that RNF216 was upregulated in human colorectal cancer (CRC) tissues and cell lines, and was associated with progression of CRC. RNF216 promoted CRC cell proliferation and migration in vitro and in vivo, largely by enhancing proteasomal degradation of BECN1, a key autophagy regulator and tumor suppressor. RNF216 restricted CRC cell autophagy through BECN1 inhibition under nutritional starvation conditions. RNF216 knockdown increased the autophagy, limiting CRC cell proliferation and migration. Moreover, BECN1 knockdown or autophagy inhibition restored proliferation and migration of RNF216-knockdown CRC cells. Collectively, our results suggested that RNF216 promoted CRC cell proliferation and migration by negatively regulating BECN1-dependent autophagy. This makes RNF216 as a potential biomarker and novel therapeutic target for inhibiting CRC development and progression.

\section{INTRODUCTION}

Globally, colorectal cancer (CRC) is one of the most common cancers with high incidence and cancer associated mortality $[1,2]$. Even in areas historically at low risk, such as China, Japan and Korea, CRC incidence is rapidly increasing in recent years $[1,3,4]$. However, CRC patient prognosis has not significantly improved, especially in cases of advanced disease. Factors predisposing patients to CRC include heredity, chronic inflammation, dietary patterns, obesity and smoking. CRC molecular mechanisms are complex, involving gene mutations, epigenetic modifications, aberrant protein ubiquitination and signaling crosstalk. Clarifying the mechanisms of CRC carcinogenesis and development will contribute to enhanced patient diagnosis and treatment.

Autophagy, a conserved mechanism maintaining cellular homeostasis, contributes to both normal and pathological processes. Improper autophagy has been associated with various diseases, including cancer [5-8]. Autophagy and autophagy-related genes are over or downregulated in cancers, which are both significantly associated with poor prognosis, suggesting the complicated biological role of autophagy in cancer [9-13]. The currently accepted hypothesis is that autophagy plays dual and contradictory roles in CRC and this appears context dependent. However, the precise mechanisms leading to CRC cell autophagy activation or inhibition are still unclear [14-17]. So exploring the mechanisms of autophagy participating in CRC and controlling autophagy properly will benefit diagnosis and current therapies of CRC.

Ring finger protein 216 (RNF216), an E3 ubiquitinprotein ligase, mediates ubiquitination and proteolytic degradation of several toll-like receptor (TLR), such as TLR4 and TLR9, restricting TLR signaling intensity and 
duration [18]. RNF216 can ubiquitinate the downstream molecules of TLRs and tumor necrosis factor (TNF) receptor 1 , such as receptor interacting serine/threonineprotein kinase 1 (RIP1), negatively regulating TNF- $\alpha$ signaling [19]. RNF216 also targets tumor necrosis factor receptor-associated factor 3 (TRAF3) for degradation, regulating RNA virus infection [20], and interacts with BECN1 in macrophages, inhibiting autophagy and impacting macrophage response to infection [21]. Thus, RNF216 plays an important role in regulating innate immunity, RNA virus infection and autophagy, all of which are closely related to $\mathrm{CRC}$ tumorigenesis. Yet, the role of RNF216 in the tumorigenesis and progression of $\mathrm{CRC}$ has been rarely clarified.

In this study, we found that RNF216 is significantly upregulated in human CRC tissues and cell lines, and is associated with disease progression. RNF216 knockdown inhibited CRC cell proliferation and migration by promoting BECN1-dependent autophagy under starvation conditions. Our work provides a potential therapeutic strategy for inhibiting CRC development and progression by targeting RNF216.

\section{RESULTS}

\section{RNF216 is upregulated in colorectal cancer}

Immunohistochemical (IHC) staining and immunoblotting analysis were used to measure RNF216 expression in 86 human CRC tissues and 7 cell lines. Table 1 shows patient clinical features and statistical analyses of IHC staining. RNF216 exhibited positive cytoplasmic staining (Figure 1A); staining was greater in cancerous lesions compared to adjacent noncancerous lesions $(P<0.001)$. RNF216 expression correlated with advanced clinical stage $(P<0.001)$ and positive lymph node metastasis $(P<0.001)$, suggesting a correlation between RNF216 expression and CRC progression (Table 1). RNF216 expression was also higher in all six CRC cell lines tested, compared with the normal colon epithelial cell line, CCD-18co (Figure 1B). We used DLD-1 and SW480 cells, two representative cell lines with high RNF216 expression, for further studies.

\section{RNF216 downregulation limits CRC cell proliferation and migration}

Because RNF216 is frequently upregulated in CRC, we knocked down RNF216 in DLD-1 and SW480 cells using a short hairpin RNA (shRNF), with scrambled shRNA as control (shNC). Knockdown efficiency was confirmed by immunoblotting (Figure 2A). RNF216 knockdown decreased proliferation and migration in both DLD-1 and SW480 cells (Figure 2B \& 2C).

To investigate the role of RNF216 in promoting CRC progression in vivo, we generated subcutaneous xenograft and liver metastasis models in nude mice using DLD-1 cells transfected with shNC and shRNF. In subcutaneous xenograft models, DLD-1-shNC tumors formed earlier and grew faster compared with the knockdown groups $(P<0.001$, Figure 2D). In liver metastasis models, RNF216 knockdown suppressed liver metastasis (Figure 2E). All together, these results showed that RNF216 promoted tumorigenesis, growth and liver metastasis in nude mice.

\section{RNF216 negatively regulates CRC cell autophagy}

RNF216 reportedly mediates ubiquitination of multiple molecules, such as TLR4 [18], RIP1 [19], TRAF3 [20], BECN1 [21], and is associated with carcinogenesis. We monitored expression of these molecules in DLD-1 and SW480 cells with or without RNF216 knockdown. Our results showed no significant changes for TLR4, RIP1 and TRAF3, while BECN1 expression was markedly increased in RNF216 knockdown cells (Figure 3A \& $\mathrm{S} 1)$. Immunoblotting showed that BECN1 expression was increased as shRNF plasmid volume increased in transfections (Figure 3B), suggesting a strong correlation between RNF216 and BECN1. The proteasome and lysosome are known to be involved in protein degradation. The ubiquitin-proteasome system involves a ubiquitinactivating enzyme, a ubiquitin-conjugating enzyme and a ubiquitin ligase (E3). The E3 ligase determines substrate specificity and most ubiquitinated proteins are then targeted for degradation by the proteasome. We treated DLD-1 cells with MG132, a proteasome inhibitor or E64d, a lysosomal inhibitor. We found that BECN1degradation was blocked by MG132 treatment, while E64d treatment did not affect RNF216-mediated BECN1 degradation (Figure 3C). These results demonstrate that RNF216 promotes BECN1 degradation through the ubiquitinproteasome pathway.

We then used IHC analysis to examine RNF216 and BECN1 expression in CRC patients. All 86 CRC cases were analyzed and scored for RNF216 and BECN1 expression. Our data showed that high RNF216 level negatively correlated with BECN1 expression (Figure $3 \mathrm{D}$, Table 2), consistent with the role of BECN1 as a tumor suppressor. Because BECN1 plays an essential role in autophagy induction [8, 11, 23-25], we subjected shNC and shRNF-transfected cells to serum starvation (normal medium without serum) and assessed autophagy by monitoring light chain 3 (LC3) via immunoblotting. Starvation increased LC3-II level, and LC3-II was significantly higher in shRNF-compared with shNCtransfected cells, implying that RNF216 restricted CRC cell autophagy during starvation (Figure 3E). Collectively, these results demonstrated that RNF216 was negatively correlated with BECN1 in CRC cells, and RNF216 knockdown restored autophagy upon starvation. 
Table 1: Correlation of RNF216 expression with clinicopathologic features in CRC

\begin{tabular}{|c|c|c|c|c|}
\hline \multirow{2}{*}{$\begin{array}{l}\text { Clinicopathologic } \\
\text { parameters }\end{array}$} & \multirow[t]{2}{*}{ Case No. } & \multicolumn{2}{|c|}{ RNF216 expression } & \multirow[t]{2}{*}{$P$ value } \\
\hline & & Low & High & \\
\hline Total cases & 86 & 45 & 41 & \\
\hline Tissue type & & & & $P<0.001$ \\
\hline $\begin{array}{l}\text { Normal colorectal } \\
\text { tissue }\end{array}$ & 86 & 86 & 0 & \\
\hline CRC tissue & 86 & 45 & 41 & \\
\hline Age & & & & $P=0.890$ \\
\hline$\leq 60$ years & 35 & 18 & 17 & \\
\hline$>60$ years & 51 & 27 & 24 & \\
\hline Gender & & & & $P=0.084$ \\
\hline Male & 43 & 18 & 25 & \\
\hline Female & 43 & 27 & 16 & \\
\hline Tumor size & & & & $P=0.665$ \\
\hline$\leq 5 \mathrm{~cm}$ & 36 & 20 & 16 & \\
\hline$>5 \mathrm{~cm}$ & 41 & 25 & 25 & \\
\hline TNM stage & & & & $P=0.019$ \\
\hline I & 12 & 9 & 3 & \\
\hline II & 28 & 19 & 9 & \\
\hline III & 24 & 10 & 14 & \\
\hline IV & 22 & 7 & 15 & \\
\hline Histologic grade & & & & $P=0.043$ \\
\hline I & 17 & 12 & 5 & \\
\hline II & 54 & 29 & 25 & \\
\hline III & 15 & 4 & 11 & \\
\hline $\begin{array}{l}\text { Lymph node } \\
\text { metastasis }\end{array}$ & & & & $P=0.009$ \\
\hline Negative & 45 & 30 & 15 & \\
\hline Positive & 41 & 15 & 26 & \\
\hline Distant metastasis & & & & $P=0.153$ \\
\hline Negative & 63 & 36 & 27 & \\
\hline Positive & 23 & 9 & 14 & \\
\hline
\end{tabular}

\section{RNF216 induces CRC cell proliferation and migration by inhibiting BECN1-mediated autophagy}

Data from our previous and current studies indicated that RNF216 inhibited autophagy by enhancing BECN1degradation, thus we explored whether RNF216 induced CRC proliferation and migration through this mechanism. BECN1 was knocked down in DLD-1 and SW480 cells by BECN1-specific siRNA or non-specific siRNA as the negative control (Figure 4A). We measured DLD-1 and SW480 cells proliferation and viability following transfection with shNC, shRNF, siBECN1 or shRNF/siBECN1 constructs, with or without 3-MA treatment (an autophagy inhibitor). RNF216 knockdown decreased proliferation and migration in both cell types 
compared with controls. In addition, this decrease was almost completely restored by BECN1 knockdown or 3-MA treatment (Figure 4B \& 4C), confirming the role of BECN1 in CRC cell proliferation and migration. These data combined showed that RNF216 promoted CRC cell proliferation and migration by negatively regulating BECN1-dependent autophagy.

\section{DISCUSSION}

RNF216 functions as an E3 ubiquitin-protein ligase and mediates ubiquitination and proteolytic degradation of TLRs and downstream molecules, restricting the intensity and duration of the innate immune response [18-20]. In addition, RNF216 inhibits TLR4-mediated autophagy in macrophages by a TLR4-independent mechanism, i.e. BECN1 ubiquitination [21]. As autophagy has been shown to affect carcinogenesis of multiple cancers, the current study showed that RNF216 is involved in CRC progression, and promotes proliferation and migration.

Increased RNF216 expression in human CRC tissues suggested its role as a potential CRC biomarker. RNF216 expression is positively associated with patients' characteristics, including advanced clinical stage, tumor size and positive lymph node metastasis, highlighting its potential for use in diagnosis and therapeutic guidance.

A
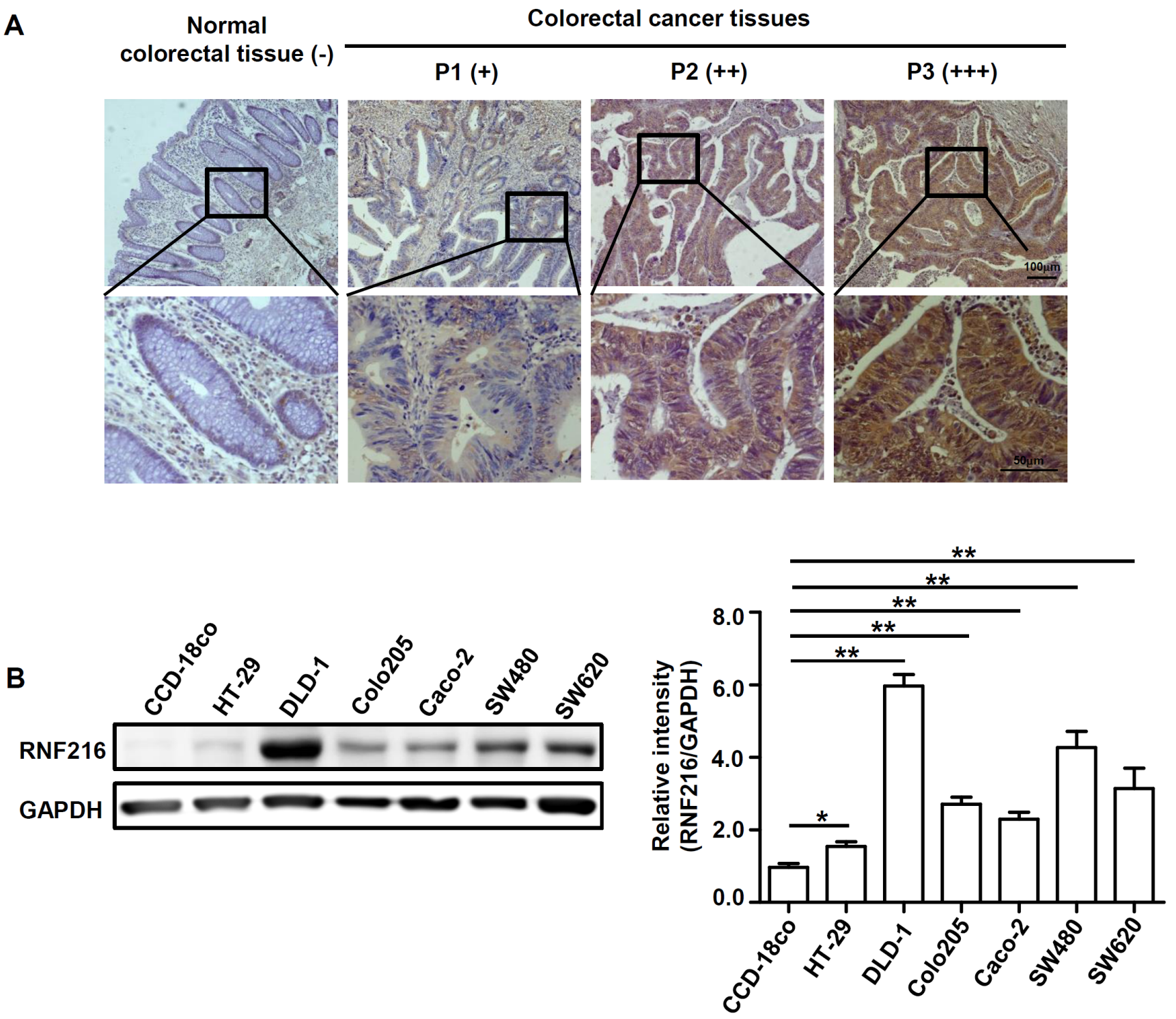

Figure 1: RNF216 expression is upregulated in human CRC tissues and cell lines. A. Human normal colorectal tissues and CRC tissues were sectioned and subjected to IHC staining against RNF216. Scale bar $=100 \mu \mathrm{m}$ (top), Scale bar $=50 \mu \mathrm{m}$ (bottom). Representative images are shown. B. CCD-18co, HT-29, DLD-1, Colo205, Caco-2, SW480 and SW620 cells were lysed and RNF216 was detected via immunoblotting. GAPDH was used as loading control. Bands were quantified using densitometry with three independent experiments. Data is shown as mean $\pm \operatorname{SEM}\left({ }^{*} P<0.05, * * P<0.001\right)$. 
Our results demonstrated that high RNF216 level promoted CRC cell proliferation and migration, while $R N F 216$ knockdown inhibited proliferation and migration, both in vitro and in vivo. Although RNF216 has several interaction partners, such as TLR4, RIP1, TRAF3 and BECN1, our data showed that only BECN1 was significantly upregulated in CRC cells in response to RNF216 knockdown, supporting that RNF216 contributes to cancer development by regulating autophagy.

Autophagy has dual and contradictory in cancer development [8, 26-29]. Autophagy could promote survival under metabolic stress and cooperatively inhibit a variety of onotherapies, including cytotoxic, targeted therapy and radiotherapy, serving to protect the genome [27, 29-31]. On the other hand, autophagy suppresses $\mathrm{CRC}$ in an earlier stage, inhibiting tumorigenesis and growth by downregulating NF- $\mathrm{kB}$ signaling, accumulating ROS, increasing DNA damage and other mechanisms [32,
33]. Moreover, monoallelic loss of the essential autophagy gene BECN1 causes susceptibility to metabolic and promotes tumorigenesis $[23,33]$, which is consistent with our observations that RNF216 could enhance BECN1 degradation dampening autophagy and contributing to cancer cells proliferation. RNF216 upregulation is one of mechanisms leading to $\mathrm{CRC}$ cell autophagy inhibition. However, there are currently no commercial compounds or small molecules specific for inhibiting RNF216. And whether there are some other potential targets of RNF216 in $\mathrm{CRC}$ development would be interesting.

BECN1 is indispensable for canonical autophagy, regulating autophagic phosphatidylinositol 3-phosphate generation and recruiting additional Atg proteins to orchestrate autophagosome formation [34]. A recent study showed that both BECN1 over- and underexpression could be related to poor colon cancer prognosis, illustrating the multifunctionality of BECN1
A

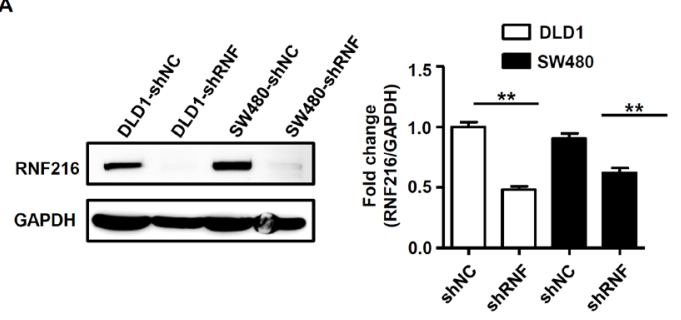

B

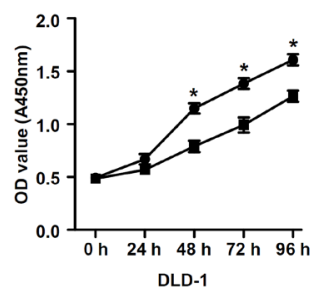

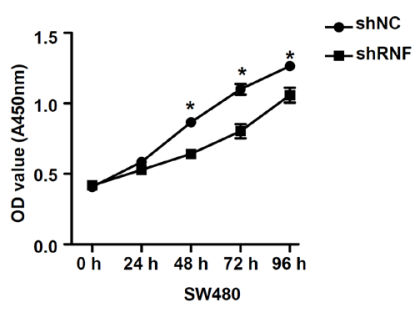

D

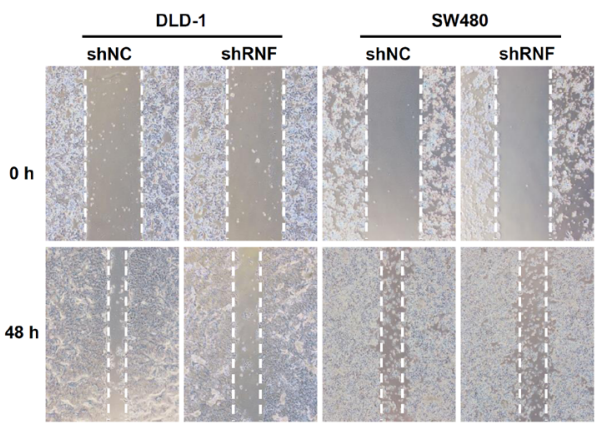

E

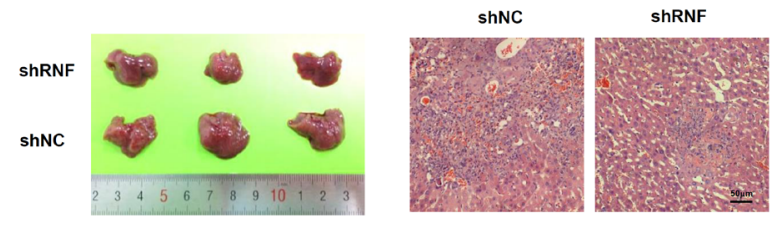

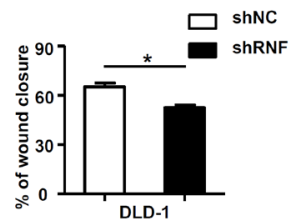
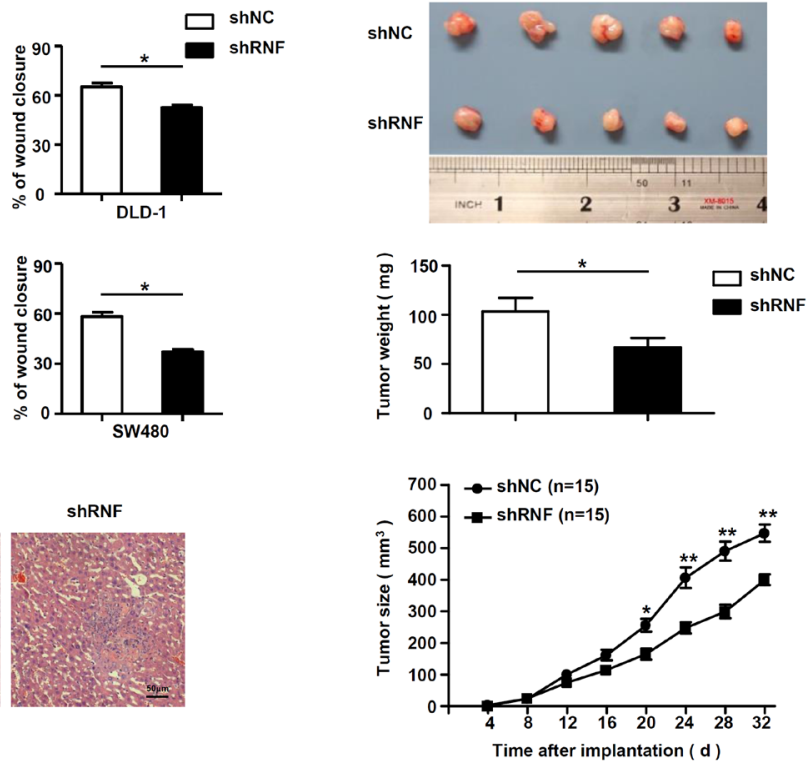

Figure 2: RNF216 promotes CRC cells proliferation and migration in vitro and in vivo. A. DLD-1 and SW480 cells were transfected with control (shNC) or RNF216-specific shRNA (shRNF). RNF216 knockdown efficiency was confirmed by immunoblotting of the endogenous protein levels. GAPDH was used as loading control. B. Cell proliferation and viability were examined at $0,24,48,72$ and $96 \mathrm{~h}$. C. Wound healing assays measured cell migration at 0 and $48 \mathrm{~h}$. D. Xenograft model in nude mice. Two different sets of nude mice were injected subcutaneously with DLD-1 DLD-1-shRNF216 or DLD-1-shNC cells. Tumors were measured every four days and removed at day 26. E. Liver metastasis model in nude mice. Images and H\&E staining of liver metastasis in nude mice injected intrasplenically with DLD-1-shRNF or DLD-1-shNC cells at three weeks post-injection. Scale bar $=50 \mu \mathrm{m}$. Representative images are shown. Data is expressed as the mean \pm SEM of three independent experiments $(* P<0.05$, $* * P<0.001)$. 
and autophagy in cancer aggressiveness [34, 35]. Our results confirmed the inhibitory role of autophagy in CRC development, consistent with other reports of BECN1mediated tumor progression inhibition.

In summary, our study found that RNF216 expression is increased in CRC tissues and correlates with clinical stage, tumor size and positive lymph node metastasis. We showed that RNF216 contributes to CRC development by promoting cell proliferation and migration via negative regulation of BECN1-dependent autophagy. Thus, RNF216 is a potential biomarker and therapeutic target for inhibiting CRC development and progression.
A

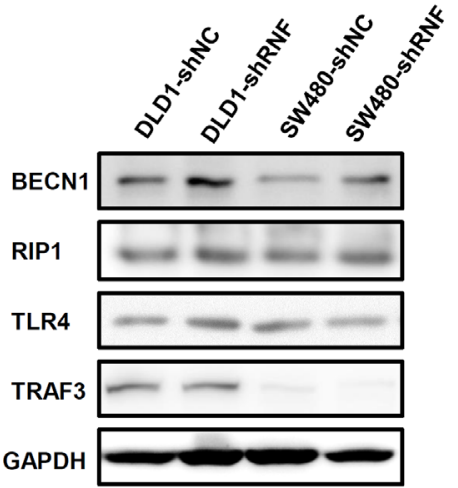

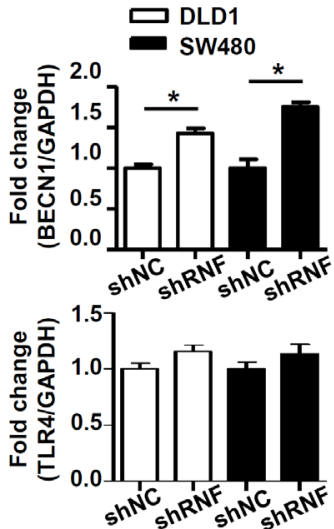

C

\begin{tabular}{l|llllll} 
ShRNF & - & - & - & + & + & + \\
E64d & - & + & - & - & + & - \\
MG132 & - & - & + & - & - & + \\
BECN1 & & & & & & \\
GAPDH
\end{tabular}

$\mathbf{E}$
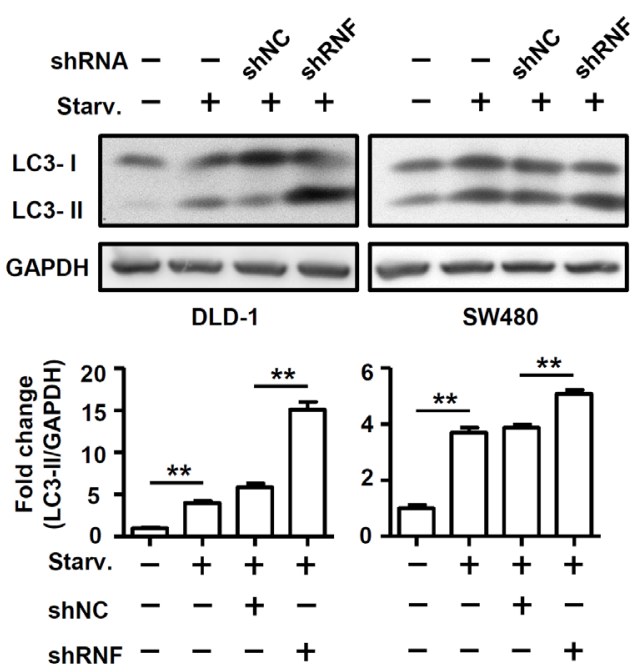

B

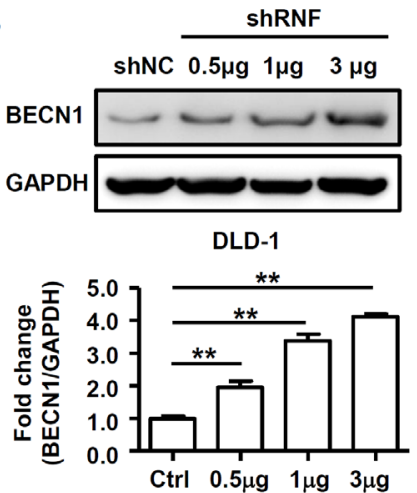

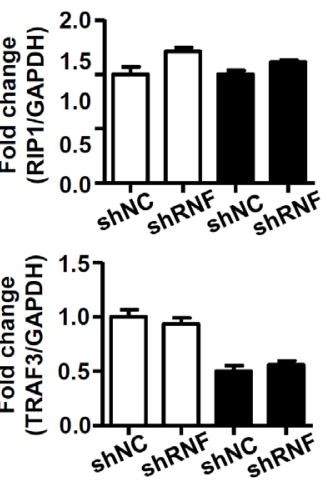

D

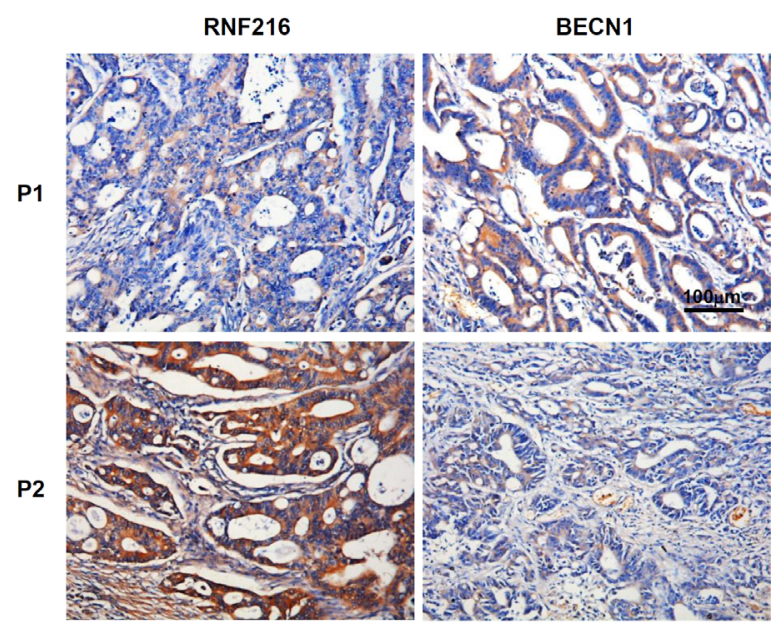

Figure 3: RNF216 promotes proteasomal degradation of BECN1 and negatively regulates CRC cell autophagy. A. DLD-1 and SW480 cells were transfected with shNC or shRNF. BECN1, RIP1, TLR4 and TRAF3 of DLD-1 and SW480 cells was done by immunoblotting. B. BECN1 was detected in DLD-1 cells transiently transfected with shNC, $0.5 \mu \mathrm{g}, 1 \mu \mathrm{g}$ and $3 \mu \mathrm{g}$ shRNF by immunoblotting. C. DLD-1 cells were treated with E64d or MG132, and BECN1 expression was measured by immunoblotting. D. RNF216 and BECN1 IHC staining in serial sections of human CRC. Examples of patients with low and high RNF216 and BECN1 expression are shown. Scale bar $=100 \mu \mathrm{m}$. E. DLD-1 and SW480 cells were subjected to serum starvation for $12 \mathrm{~h}$. Autophagy was assessed by immunoblotting for LC3. Data are expressed as the mean \pm SEM of three independent experiments $\left({ }^{*} P<0.05, * * P<0.001\right)$. GAPDH was used as a loading control. 
Table 2: The relationship between RNF216 expression and BECN1 in human CRC tissues

\begin{tabular}{ccccc}
\hline & & \multicolumn{2}{c}{ RNF216 expression } & \multicolumn{2}{c}{$\begin{array}{c}\text { Correlation coefficient } \\
(\mathbf{R})\end{array}$} & High & Low & 20 & $\mathbf{R}=\mathbf{- 0 . 2 7 8}(P=\mathbf{0 . 0 0 9 9})$ \\
\cline { 3 - 5 } BECN1 & Low & 10 & 21 & 35 \\
\hline
\end{tabular}

A

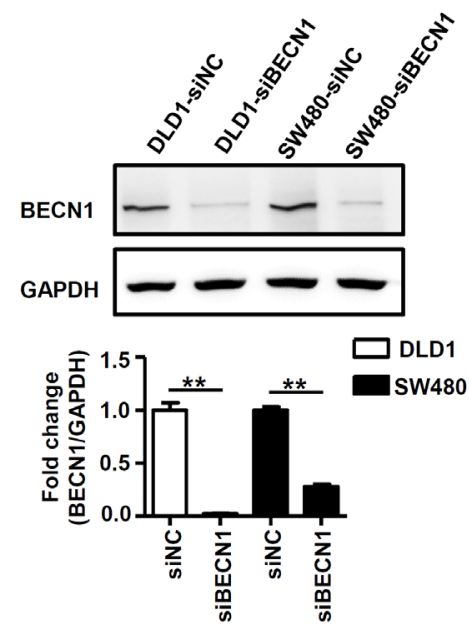

B
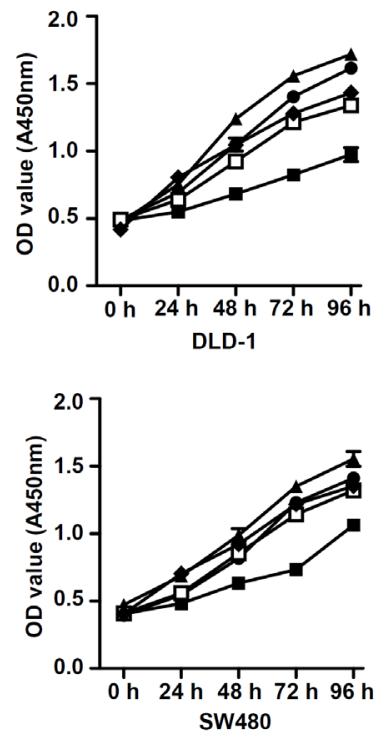

C
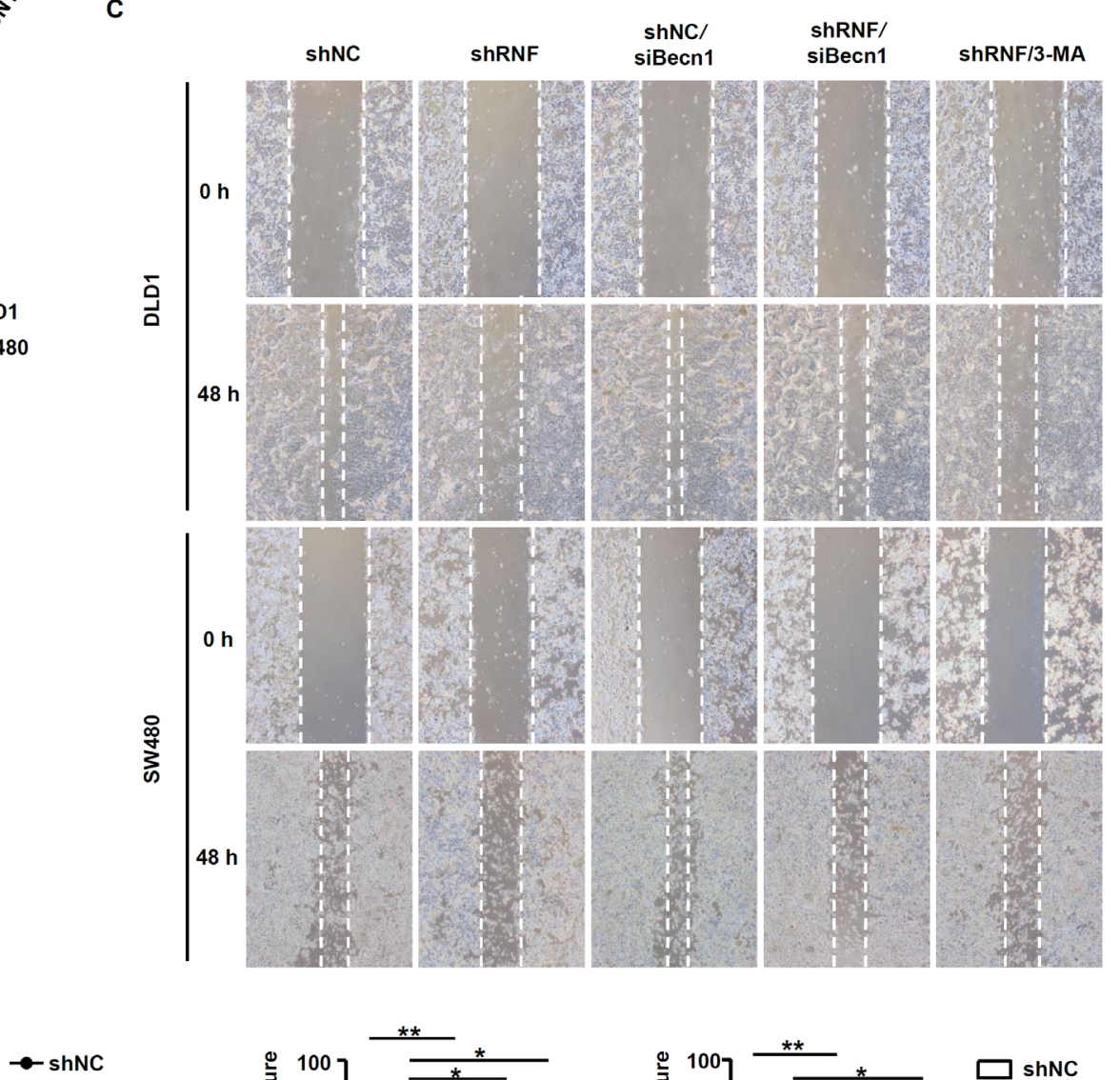

$\rightarrow$ shRNF

\pm shNC/siBecn1

$-\square-$ shRNF/siBecn1

$\rightarrow$ shRNF/3-MA

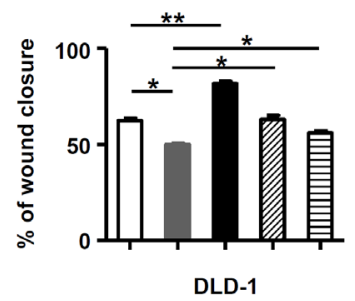

$\square$ shNC

shRNF

shNC/siBecn1

DhRNF/siBecn1

可 shRNF/3-MA

Figure 4: RNF216 induces proliferation and migration via BECN1 ubiquitination and inhibition of autophagy. A. BECN1 was detected in siNC and siBECN1 transfected DLD-1 and SW480 cells w by immunoblotting. GAPDH was used as loading control. B. WST-1 assay was used to detect proliferation and viability in DLD-1 and SW480 cells transfected with shNC, shRNF, siBECN1 or shRNF/siBECN1, or treated with 3-MA $(8 \mu \mathrm{M})$ at $0,24,48,72$ and $96 \mathrm{~h}$. C. Wound healing assay was used to detect migration in DLD-1 and SW480 cells transfected with shNC, shRNF, siBECN1 or shRNF/siBECN1, or treated with 3-MA at 0 and $48 \mathrm{~h}$. Data are expressed as the mean \pm SEM of three independent experiments $(* P<0.05, * * P<0.001)$. 


\section{MATERIALS AND METHODS}

\section{Patients and specimens}

This study was approved by the Biomedical Research Ethics Committee of Shanghai Institutes for Biological Sciences, Chinese Academy of Sciences. All patients provided full consent for the present study. CRC samples were collected from 86 patients undergoing curative-intent surgery at the Department of Surgery, Renji Hospital, Shanghai Jiao Tong University School of Medicine between 2008 and 2011. Additionally, 86 normal colorectal tissues adjacent to colorectal cancer tissues were collected. None of the patients had received any preoperative treatment. All specimens were reviewed by two clinical pathologists to verify histologic diagnoses. Cancers were staged according to the classification of the American Joint Committee on Cancer pathologic tumornode-metastasis (TNM).

\section{Cell culture}

Human colorectal carcinoma cell lines (HT-29, DLD-1, Colo205, Caco-2, SW480 and SW620) and one normal colon epithelial cell line (CCD-18Co) used in this study were purchased from the American type culture collection (ATCC, Manassas, VA, USA). Cells were cultured in RPMI 1640, DMEM or L-15 (Gibco, Grand Island, NY, USA) medium supplemented with $10 \%$ fetal bovine serum, $100 \mathrm{U} / \mathrm{mL}$ penicillin and 100 $\mathrm{mg} / \mathrm{mL}$ streptomycin (Gibco, Grand Island, NY, USA) in humidified air at $37^{\circ} \mathrm{C}$ with $5 \% \mathrm{CO} 2$.

\section{Immunohistochemistry assay and the quantification}

Sections were subjected to deparaffination, rehydration, antigen retrieval and blockade of nonspecific bindings. The specimens were incubated with primary anti-human RNF216 (Abcam, Cambridge, UK) and BECN1 (Cell Signaling Technology, Danvers, MA, USA) antibodies and second antibody, and then counterstained with hematoxylin and mounted. Immunostaining was independently examined by two clinical pathologists who were unaware of the patient outcome. For each sample, five fields $(100 \times)$ were randomly selected. Staining intensity and percentage of positive tumor cells were assessed. Staining was quantified as described previously [22].

\section{Lentiviral vector construction and transfection}

The following oligonucleotides were inserted into the lentiviral vector, pLVX-shRNA2, RNF216 (shRNA): 5'-GGACACTATGCAATCACCCG-3' and scrambled control (shNC): 5'-AGGACTGAGTGTACCGTCT-3' (Genepharma, Shanghai, China). High titer lentiviral stocks were produced to infect CRC cells, and transfection efficiency exceeded $95 \%$.

\section{siRNA transfection}

Cells were cultured for $24 \mathrm{~h}$ prior to transient transfection with BECN1 siRNA, RNF216 siRNA or negative control siRNA (Life Technologies, Grand Island, NY, USA) using Lipofectamine 3000 Transfection Reagent (Invitrogen, Carlsbad, CA, USA) according to the manufacturer's instructions. After $48 \mathrm{~h}$, transfected cells were harvested for immunoblotting and subsequent assays.

\section{Immunoblotting}

Cell lysates were subjected to SDS-PAGE and transferred to polyvinylidene difluoride membranes. Target proteins were detected with primary antibodies against LC3 (Cell Signaling Technology, Danvers, MA, USA), BECN1 (Santa Cruz Biotechnology, Santa Cruz, CA, USA), RNF216 (Abcam, Cambridge, UK), HRP-conjugated anti-mouse and anti-rabbit secondary antibodies (Jackson ImmunoResearch, West Grove, PA, USA). Bands were visualized using Chemilucent Plus Western Blot Kit (Millipore, Darmstadt, Germany). Bands densitometry was quantified by ImageJ software (National Institutes of Health, Bethesda, MD, USA).

\section{Cell proliferation assay}

DLD-1 and SW480 cell lines were seeded in 96well plate at 3000 cells/well in $100 \mu \mathrm{L}$ medium. WST-1 assay (Roche, Basel, Switzerland) was used to measure viable proliferating cells at $0,24,48,72$ and $96 \mathrm{~h}$ after plating. Absorbance was measured at $450 \mathrm{~nm}$ using a spectrophotometer.

\section{Wound healing assay}

DLD-1 and SW480 cell lines were seeded in 6-well plates at $>90 \%$ confluency. A wound was created with a sterile $200 \mu \mathrm{L}$ pipet tip. Cells were rinsed with PBS and 1.5 $\mathrm{mL}$ of low serum medium was added to the wells. Pictures were taken at $0,6,12,24$ and $48 \mathrm{~h}$.

\section{Animal study}

Six-week old BALB/c nude mice were used and treated under specific pathogen-free conditions. To establish CRC xenografts, mice were injected subcutaneously with $5 \times 10^{6}$ DLD- 1 cells in PBS ( $\mathrm{n}=15$ per group). Mice were examined every four days and scored according to tumor width and length. Tumor volume was estimated by the formula width ${ }^{2} \times$ length $\times 0.52 \mathrm{in}^{3}$. Tumors were removed on day 26 . To establish a liver metastasis model, control and RNF216 knockdown cells were injected into the spleen $\left(5 \times 10^{6}\right.$ per mouse $)$ of nude 
mice ( $n=15$ per group). Mice were euthanized and livers were removed on day 21 . H\&E staining was performed on paraffin embedded liver sections. All animal procedures were approved by the Animal Welfare \& Ethics Committee of Shanghai Jiao Tong University School of Medicine.

\section{Statistical analysis}

Differences were evaluated using Statistical Package for Social Science software (version 20.0, SPSS Inc., Chicago, USA). Significant differences were evaluated using an independent-samples $t$ test or Wilcoxon rank test, except that multiple treatment groups were compared within individual experiments by ANOVA. The association of staining intensity with clinicopathologic patterns was assessed with Chi-square test and two-sided Fisher's exact test to assess significant differences. Correlation between RNF216 and BECN1 expression were analyzed via Chisquare test. $P<0.05$ was considered significant. All values were presented as mean \pm S.E.M.

\section{CONFLICTS OF INTEREST}

The authors declare no conflicts of interest.

\section{GRANT SUPPORT}

This study was supported by the Ministry of Science and Technology of China (2015CB943303, 2014CB943303), National Natural Science Foundation of China (81502607, 81502506, 31570905, 31300708 and 81471217), the Program of Science and Technology Commission of Shanghai Municipality (15JC1402900, 13ZR1423200 and 14ZR1446300).

\section{REFERENCES}

1. Jemal A, Bray F, Center MM, Ferlay J, Ward E, Forman D. Global cancer statistics. CA Cancer J Clin. 2011; 61:69-90.

2. Jemal A, Siegel R, Ward E, Hao Y, Xu J, Murray T, Thun MJ. Cancer statistics, 2008. CA Cancer J Clin. 2008; 58:71-96.

3. Sung JJ, Lau JY, Young GP, Sano Y, Chiu HM, Byeon JS, Yeoh KG, Goh KL, Sollano J, Rerknimitr R, Matsuda $\mathrm{T}, \mathrm{Wu} \mathrm{KC}, \mathrm{Ng} \mathrm{S}$, et al. Asia Pacific consensus recommendations for colorectal cancer screening. Gut. 2008; 57:1166-1176.

4. Sung JJ, Ng SC, Chan FK, Chiu HM, Kim HS, Matsuda T, Ng SS, Lau JY, Zheng S, Adler S, Reddy N, Yeoh KG, Tsoi KK, et al. An updated Asia Pacific Consensus Recommendations on colorectal cancer screening. Gut. 2015; 64:121-132.

5. Cappelletti C, Galbardi B, Kapetis D, Vattemi G, Guglielmi V, Tonin P, Salerno F, Morandi L, Tomelleri G, Mantegazza R, Bernasconi P. Autophagy, inflammation and innate immunity in inflammatory myopathies. PLoS One. 2014; 9:e111490.

6. He C, Klionsky DJ. Regulation mechanisms and signaling pathways of autophagy. Annu Rev Genet. 2009; 43:67-93.

7. Levine B, Mizushima N, Virgin HW. Autophagy in immunity and inflammation. Nature. 2011; 469:323-335.

8. Kimmelman AC. The dynamic nature of autophagy in cancer. Genes Dev. 2011; 25:1999-2010.

9. Giatromanolaki A, Sivridis E, Mendrinos S, Koutsopoulos AV, Koukourakis MI. Autophagy proteins in prostate cancer: relation with anaerobic metabolism and Gleason score. Urol Oncol. 2014; 32:39 e11-38.

10. Zhang W, Li Q, Song C, Lao L. Knockdown of autophagyrelated protein 6, Beclin-1, decreases cell growth, invasion, and metastasis and has a positive effect on chemotherapyinduced cytotoxicity in osteosarcoma cells. Tumour Biol. 2015; 36:2531-2539.

11. Yue Z, Jin S, Yang C, Levine AJ, Heintz N. Beclin 1, an autophagy gene essential for early embryonic development, is a haploinsufficient tumor suppressor. Proc Natl Acad Sci U S A. 2003; 100:15077-15082.

12. Weng J, Wang C, Wang Y, Tang H, Liang J, Liu X, Huang $\mathrm{H}$, Hou J. Beclin1 inhibits proliferation, migration and invasion in tongue squamous cell carcinoma cell lines. Oral Oncol. 2014; 50:983-990.

13. Liu XD, Yao J, Tripathi DN, Ding Z, Xu Y, Sun M, Zhang J, Bai S, German P, Hoang A, Zhou L, Jonasch D, Zhang $\mathrm{X}$, et al. Autophagy mediates HIF2alpha degradation and suppresses renal tumorigenesis. Oncogene. 2015; 34:2450-2460.

14. Vermeulen L, De Sousa EMF, van der Heijden M, Cameron K, de Jong JH, Borovski T, Tuynman JB, Todaro M, Merz C, Rodermond H, Sprick MR, Kemper K, Richel DJ, Stassi G, Medema JP. Wnt activity defines colon cancer stem cells and is regulated by the microenvironment. Nat Cell Biol. 2010; 12:468-476.

15. Chen N, Karantza-Wadsworth V. Role and regulation of autophagy in cancer. Biochim Biophys Acta. 2009; 1793:1516-1523.

16. Lai $\mathrm{K}$, Killingsworth $\mathrm{MC}$, Lee $\mathrm{CS}$. The significance of autophagy in colorectal cancer pathogenesis and implications for therapy. J Clin Pathol. 2014; 67:854-858.

17. Burada F, Nicoli ER, Ciurea ME, Uscatu DC, Ioana M, Gheonea DI. Autophagy in colorectal cancer: An important switch from physiology to pathology. World J Gastrointest Oncol. 2015; 7:271-284.

18. Chuang TH, Ulevitch RJ. Triad3A, an E3 ubiquitin-protein ligase regulating Toll-like receptors. Nat Immunol. 2004; 5:495-502.

19. Fearns C, Pan Q, Mathison JC, Chuang TH. Triad3A regulates ubiquitination and proteasomal degradation of RIP1 following disruption of Hsp90 binding. J Biol Chem. 2006; 281:34592-34600. 
20. Nakhaei P, Mesplede T, Solis M, Sun Q, Zhao T, Yang L, Chuang TH, Ware CF, Lin R, Hiscott J. The E3 ubiquitin ligase Triad3A negatively regulates the RIG-I/MAVS signaling pathway by targeting TRAF3 for degradation. PLoS Pathog. 2009; 5:e1000650.

21. Xu C, Feng K, Zhao X, Huang S, Cheng Y, Qian L, Wang Y, Sun H, Jin M, Chuang TH, Zhang Y. Regulation of autophagy by E3 ubiquitin ligase RNF216 through BECN1 ubiquitination. Autophagy. 2014; 10:2239-2250.

22. Wu Q, Wang H, Zhao X, Shi Y, Jin M, Wan B, Xu H, Cheng Y, Ge H, Zhang Y. Identification of G-proteincoupled receptor 120 as a tumor-promoting receptor that induces angiogenesis and migration in human colorectal carcinoma. Oncogene. 2013; 32:5541-5550.

23. Liang XH, Jackson S, Seaman M, Brown K, Kempkes B, Hibshoosh H, Levine B. Induction of autophagy and inhibition of tumorigenesis by beclin 1. Nature. 1999; 402:672-676

24. Cianfanelli V, D'Orazio M, Cecconi F. AMBRA1 and BECLIN 1 interplay in the crosstalk between autophagy and cell proliferation. Cell Cycle. 2015; 14:959-963.

25. Kang R, Zeh HJ, Lotze MT, Tang D. The Beclin 1 network regulates autophagy and apoptosis. Cell Death Differ. 2011; 18:571-580.

26. Bhutia SK, Dash R, Das SK, Azab B, Su ZZ, Lee SG, Grant S, Yacoub A, Dent P, Curiel DT, Sarkar D, Fisher PB. Mechanism of autophagy to apoptosis switch triggered in prostate cancer cells by antitumor cytokine melanoma differentiation-associated gene 7/interleukin-24. Cancer Res. 2010; 70:3667-3676.

27. Chen YJ, Chi CW, Su WC, Huang HL. Lapatinib induces autophagic cell death and inhibits growth of human hepatocellular carcinoma. Oncotarget. 2014; 5:4845-4854. doi: 10.18632/oncotarget.2045.
28. Chiao MT, Cheng WY, Yang YC, Shen CC, Ko JL. Suberoylanilide hydroxamic acid (SAHA) causes tumor growth slowdown and triggers autophagy in glioblastoma stem cells. Autophagy. 2013; 9:1509-1526.

29. Choi JH, Cho YS, Ko YH, Hong SU, Park JH, Lee MA. Absence of autophagy-related proteins expression is associated with poor prognosis in patients with colorectal adenocarcinoma. Gastroenterol Res Pract. 2014; 2014:179586.

30. Cheng P, Ni Z, Dai X, Wang B, Ding W, Rae Smith A, $\mathrm{Xu}$ L, Wu D, He F, Lian J. The novel BH-3 mimetic apogossypolone induces Beclin-1- and ROS-mediated autophagy in human hepatocellular carcinoma [corrected] cells. Cell Death Dis. 2013; 4:e489.

31. Dalby KN, Tekedereli I, Lopez-Berestein G, Ozpolat B. Targeting the prodeath and prosurvival functions of autophagy as novel therapeutic strategies in cancer. Autophagy. 2010; 6:322-329.

32. Komatsu M, Kurokawa H, Waguri S, Taguchi K, Kobayashi A, Ichimura Y, Sou YS, Ueno I, Sakamoto A, Tong KI, Kim M, Nishito Y, Iemura S, et al. The selective autophagy substrate p62 activates the stress responsive transcription factor Nrf2 through inactivation of Keap1. Nat Cell Biol. 2010; 12:213-223.

33. Mathew R, Kongara S, Beaudoin B, Karp CM, Bray K, Degenhardt K, Chen G, Jin S, White E. Autophagy suppresses tumor progression by limiting chromosomal instability. Genes Dev. 2007; 21:1367-1381.

34. Wirawan E, Lippens S, Vanden Berghe T, Romagnoli A, Fimia GM, Piacentini M, Vandenabeele P. Beclin1: a role in membrane dynamics and beyond. Autophagy. 2012; 8:6-17.

35. Groulx JF, Khalfaoui T, Benoit YD, Bernatchez G, Carrier JC, Basora N, Beaulieu JF. Autophagy is active in normal colon mucosa. Autophagy. 2012; 8:893-902. 